\title{
A MENSURAÇÃO DO ESTIGMA INTERNALIZADO: REVISÃO SISTEMÁTICA DA LITERATURA ${ }^{1}$
}

\author{
Rhaisa Gontijo Soares \\ Fabricia Creton Nery \\ Pollyanna Santos Silveira \\ Ana Regina Noto ${ }^{\Phi}$ \\ Telmo Mota Ronzani ${ }^{\theta}$
}

\begin{abstract}
RESUMO. O estigma internalizado é um fenômeno processual que se estabelece à medida que o indivíduo torna-se consciente de sua condição de estigmatizado, concorda e aplica a si próprio os estereótipos negativos sobre seu transtorno. Com o objetivo de conhecer os instrumentos de mensuração desse fenômeno, realizou-se, em janeiro de 2011, uma revisão sistemática da literatura nas bases de dados Web of Science, Pubmed, Psycinfo, Lilacs e Scielo, utilizando-se os termos internalized stigma, self-stigma, estigma internalizado e autoestigma. Onze escalas foram analisadas, seis das quais mensuravam estigma internalizado referente a transtornos mentais. A análise revelou problemas conceituais e metodológicos dos estudos, apontando dificuldades intrínsecas à investigação do fenômeno e fornecendo subsídios para pesquisas futuras. Apesar das limitações, o crescente número de escalas demonstra a relevância do tema no campo da Saúde Mental e proporciona um avanço do conhecimento acerca dos fatores envolvidos no processo de estigmatização.
\end{abstract}

Palavras-chave: estigma; psicometria; pesquisa bibliográfica.

\section{THE MEASUREMENT OF INTERNALIZED STIGMA: SYSTEMATIC REVIEW OF LITERATURE}

\begin{abstract}
Internalized stigma is a processual phenomenon established as the individual becomes aware of his stigmatized condition, and agrees to apply its own negative stereotypes about their disorder. Aiming to cognize the measurement instruments of internalized stigma, a systematic review of literature was undertaken in January 2011 on Web of Science, PubMed, PsycINFO, Lilacs and Scielo databases, using the terms internalized stigma and self-stigma. Eleven scales were analyzed, 6 of them measured internalized stigma toward mental disorders. The analysis reveals conceptual and methodological problems of the studies, pointing out intrinsical difficulties to investigate the phenomenon and offering subsidies to forthcoming researches. In spite of the limitations, the growing number of scales demonstrates the relevance of the topic in Mental Health's area and provides an advance in knowledge about the factors related to the stigmatization process.
\end{abstract}

Key words: Stigma; psychometry; bibliographical research.

\section{LA MENSURACIÓN DEL ESTIGMA INTERNALIZADO: REVISIÓN SISTEMÁTICA DE LA LITERATURA}

RESUMEN. Estigma internalizado es un fenómeno procesal establecido a la medida en que el individuo reconoce su condición estigmatizada, concuerda con ella y aplica a si mismo los estereotipos negativos cerca de su trastorno. Con

\footnotetext{
Apoio: Capes, CNPq, Fapemig.

Mestre em Psicologia pela Universidade Federal de Juiz de Fora. Auxliar de pesquisa da Universidade Federal de Juiz de Fora, Brasil.

If Mestranda em Psicologia pela Universidade Federal de Juiz de Fora.Auxiliar de pesquisa da Universidade Federal de Juiz de Fora, Brasil.

æ Mestre em Psicologia pela Universidade Federal de Juiz de Fora. Doutoranda em Psicologia pela Universidade Federal de São Paulo, Brasil.

$\Phi$ Doutora em Psicobiologia pela Universidade Federal de São Paulo. Professora adjunta da Universidade Federal de São Paulo, Brasil.

$\theta \quad$ Doutor em Psicobiologia pela Universidade Federal de São Paulo. Professor adjunto da Universidade Federal de Juiz de Fora , Brasil. Bolsista de produtividade CNPq.
} 
objetivo de conocer los instrumentos de mensuración del fenómeno, se ha realizado, en enero 2011, una revisión sistemática de la literatura en las bases de datos Web of Science, Pubmed, Psycinfo, Lilacs y Scielo, utilizando los términos internalized stigma, self-stigma, estigma internalizado y auto-estigma. Se han analizado once escalas, de las cuales 6 mensuraban estigma internalizado relacionado a los trastornos mentales. El análisis ha revelado problemas conceptuales y metodológicos de los estudios, indicando dificultades intrínsecas a la investigación del fenómeno y suministrando subsidios para pesquisas futuras. No obstante las limitaciones, el creciente número de escalas demuestra la relevancia del tema en el campo de la Salud Mental, y proporciona un avance del conocimiento cerca de los factores envueltos en el proceso de estigmatización.

Palabras-clave: Estigma; psicometría; investigación bibliografica.

O processo de estigmatização tem sido apontado como profundamente danoso a algumas condições de saúde estigmatizadas na cultura atual (Fung, Tsang, Corrigan, Lam, \& Cheng, 2007; Kalichman et al., 2009; Kanter, Rusch, \& Brondino, 2008; Ritsher, Otilingam, \& Grajales, 2003; Vogel, Wade, \& Haake, 2006); no entanto, alguns estudos ainda sustentam que o estigma não apresenta efeitos negativos no indivíduo (Crocker \& Lawrence, 1999; Crocker, \& Major, 1989). Segundo Barney, Griffiths, Jorm e Christensen (2006), essa contradição quanto aos efeitos da estigmatização pode resultar da complexidade do conceito "estigma".

Apesar de sua raiz sociológica - em que o estigma é descrito como um processo inerente à interação social e caracterizado por categorias como rotulação, status social, "desviante" e "normal" (Goffman, 1963) - definições recentes têm se aproximado mais de perspectivas psicológicas, destacando processos psicossociais e privilegiando componentes como crenças, estereótipos, atribuição de causalidade, atitudes, preconceito e discriminação (Link, Phelan, Bresnahan, Stueve, \& Pescosolido, 1999; Link, \& Phelan, 2001). Destarte a vertente psicossocial tem focado a natureza contextual e dinâmica do estigma, enfatizando os efeitos imediatos do contexto social e situacional na perspectiva do estigmatizador, do estigmatizado e da interação entre ambos (Corrigan, 1998).

De acordo com Corrigan e Watson (2002), existem dois tipos de estigma: o estigma público e o autoestigma. O estigma público é a percepção de um grupo ou da sociedade de que um indivíduo é socialmente inaceitável, devido a características físicas ou pessoais muitas vezes associadas a reações negativas e, consequentemente, à desvalorização do indivíduo. Já o estigma internalizado ocorre à medida que o indivíduo em condição estigmatizante torna-se consciente dos estereótipos negativos sobre seu transtorno, concorda com eles e os aplica a si próprio (Corrigan \& Watson, 2002).

Por conseguinte, o processo de internalização do estigma torna-se central para as condições psicológicas destes indivíduos, acarretando, por exemplo, diminuição de autoestima e autoeficácia, percepção de descrédito, sentimentos de vergonha, culpa, angústia, raiva ou autorreprovação (Corrigan, 1998; Corrigan, Watson, \& Barr, 2006). Tal processo também gera várias implicações práticas na vida de um paciente, entre as quais perspectivas limitadas de recuperação e restrição de redes sociais (Corrigan, 1998; Link, Mirotznik, \& Cullen, 1991; Link, Struening, Neese-Todd, Asmussen, \& Phelan, 2001). Estes fatores podem levar ao isolamento, ao desemprego e ao baixo rendimento, influenciando na disposição dos sujeitos a procurar tratamento, pois, na tentativa de evitar que sua condição de saúde se torne pública, na maioria das vezes eles não o procuram (Kanter, Rusch, \& Brondino, 2008). Neste sentido, muitas vezes a condição de estigmatização pode se tornar muito mais danosa do que o próprio transtorno (Ronzani, Furtado, \& Higgins-Biddle, 2009).

Considera-se importante, diante da magnitude dos efeitos da estigmatização, ressaltar a necessidade de desenvolver iniciativas que instaurem, em relação ao estigma, intervenções que sejam efetivas e possam ajudar os pacientes a reduzir sua internalização e aumentar a adesão ao tratamento. Não obstante, existem algumas questões que ainda são consideradas um desafio aos pesquisadores no tocante ao entendimento do estigma, como, por exemplo: compreender os resultados da relação entre estigma e busca por tratamento; desenvolver e avaliar a efetividade de programas relativos ao estigma que aumentem a procura por tratamento; e construir medidas válidas e confiáveis de estigma que sejam baseadas em um modelo científico, teórico e empírico deste conceito (Kanter et al., 2008) e possam abranger o crescente interesse pela questão em diferentes áreas.

Mesmo sendo um desafio, estudos indicam que a construção/validação de instrumentos capazes de mensurar o estigma internalizado proporciona um avanço do conhecimento, pois esses instrumentos investigam fatores envolvidos no processo, como, por exemplo, os mecanismos prejudiciais causados pelo transtorno e o impacto na vida do sujeito, 
impulsionando estratégias de redução do estigma para a obtenção de melhores resultados no tratamento de saúde (Fung et al., 2007; Kanter et al., 2008; Luoma, Kohlenberg, Hayes, Bunting, \& Rye, 2008; Ritsher et al., 2003).

Até o fim da década de 90 as escalas de estigma comumente utilizadas na literatura eram destinadas a medir as atitudes de um público geral acerca dos transtornos mentais, ou a avaliar experiências passadas a respeito do estigma e da discriminação (Link et al., 1991). Somente nos últimos dez anos é que se observou um aumento na construção de escalas sobre o estigma internalizado (Brohan, Slade, Clement, \& Thornicroft, 2010).

Assim, o objetivo do presente artigo foi realizar uma revisão sistemática da literatura para conhecer os instrumentos de mensuração de estigma internalizado e apreender como esses estudos foram realizados.

\section{MÉTODO}

A busca foi realizada em janeiro de 2011 nas bases de dados Web of Science, Pubmed, Psycinfo, Lilacs e Scielo. Os termos utilizados nas bases Web of Science, Pubmed, Psycinfo e Lilacs foram "internalized stigma" e "self-stigma", e na base Scielo foram utilizados "estigma internalizado" e "autoestigma". Para melhor especificidade dos termos de busca nas bases selecionadas, não foram utilizadas palavras-chaves indexadas pelas próprias bases. Definidos os descritores bibliográficos, iniciou-se o processo de busca dos artigos. Apesar de a busca terse limitado a artigos de revistas (journal article), a pesquisa abrangeu todos os anos desde a data da primeira publicação encontrada sobre o tema até janeiro de 2011.

Foram encontrados, ao todo, 501 estudos catalogados, dos quais 184 estavam na Web of Science, 134 na Pubmed, 183 na Psycinfo e 0 (zero) na Lilacs e Scielo. Do total referido acima, 229 artigos estavam duplicados, por isso foram excluídos da análise, restando, então, 272 estudos finais. Destes, foram incluídos aqueles que preenchiam os seguintes critérios: a) ser estudo empírico (como delineamento do tipo de pesquisa); b) ser pesquisa com seres humanos; c) ser estudo de construção e/ou validação de escalas que mensurasse especificamente o estigma internalizado. Os critérios de exclusão foram: a) Não apresentar resumo do estudo na base de dados; e b) ser artigo publicado em outros idiomas que não o inglês, o espanhol ou o português.

Após a leitura dos 272 resumos, verificou-se que 249 estudos não estavam voltados à construção e validação de escalas relacionadas ao estigma, restando apenas 23. Finalmente, apenas doze estudos mensuravam especificamente o estigma internalizado, atendendo aos critérios de inclusão da pesquisa. Como estratégia de coleta dos textos completos, optou-se por utilizar os recursos do portal de periódicos da Capes e solicitação direta por e-mail aos autores. Nesta etapa, mais um estudo foi excluído, uma vez que, apesar de seu resumo estar em língua inglesa, o artigo completo estava escrito em chinês.

Assim, a partir da leitura dos onze artigos, realizou-se sua tabulação com o propósito de identificar os principais indicadores bibliométricos das produções e as principais características e propriedades psicométricas das escalas. Foram delimitados como categorias para análise bibliométrica: autores; ano, país e idioma de publicação; país onde foi realizado o estudo; periódico; principal objetivo do estudo (construção ou validação de instrumento); e o público para o qual a escala foi construída/validada.

Finalmente, realizou-se a análise descritiva das definições do conceito de estigma internalizado utilizadas pelos autores, das principais justificativas apresentadas pelos autores para a construção/validação das escalas, dos aspectos metodológicos dos estudos, dos procedimentos utilizados pelos autores na construção e validação das escalas e das propriedades psicométricas das escalas.

\section{RESULTADOS}

\section{Indicadores bibliométricos}

Em relação à sua autoria, os artigos seriam categorizados em autoria múltipla e autoria única, porém todos os artigos apresentaram autoria múltipla, variando de dois a sete autores. Apenas um autor, Patrick Corrigan, participou de duas publicações, enquanto 44 autores fizeram apenas uma publicação sobre o tema.

A primeira publicação sobre o tema data do ano de 2003, em que houve apenas uma publicação. Foram publicados dois artigos por ano em 2006, 2007, 2008, 2009 e 2010, não havendo publicações nos anos de 2004 e 2005.

No que se refere ao país de publicação dos artigos, a maior parte $(n=7)$ foi publicada nos Estados Unidos da América (EUA), seguindo-se a Inglaterra $(\mathrm{n}=2)$, a Turquia e a Austrália com uma publicação cada. Em relação ao idioma utilizado nas publicações, todos os 11 artigos analisados foram publicados em língua inglesa. 
Quanto ao país de realização do estudo, oito trabalhos foram desenvolvidos nos EUA. Destes, sete estudos apresentaram amostra composta somente por pessoas dos EUA, e um incluiu, concomitantemente, pessoas dos EUA, da África do Sul e da Suazilândia. Em adição, um estudo foi realizado na Turquia, um na China e, por fim, um na Austrália. No que diz respeito ao veículo de divulgação dos artigos, nenhum estudo teve publicação na mesma revista científica.

Apenas dois estudos tinham como principal objetivo a validação de escalas de estigma internalizado, enquanto os outros nove objetivavam a construção de escalas de estigma internalizado. As escalas foram construídas/validadas para diferentes populações, a maior parte $(n=6)$ para pessoas com transtornos mentais, das quais duas eram destinadas especificamente a pacientes com depressão, enquanto as outras quatro destinavam-se a pacientes com transtornos mentais gerais. Dos cinco estudos restantes, duas escalas foram construídas para avaliar o estigma internalizado em pessoas com HIV/AIDS. Uma escala foi construída para cada uma das seguintes condições: obesidade, pessoas com desordens neurológicas e busca por ajuda psicológica.

\section{Definição do conceito de estigma internalizado}

No que diz respeito ao conceito de estigma internalizado, houve pouca variação dos autores citados, consequentemente, pouca alteração em sua definição, uma vez que todas as definições utilizadas se referiram ao conceito como um constructo que traz consequências negativas para o indivíduo estigmatizado. Os dois estudos de construção de escalas de estigma internalizado para pessoas com HIV/AIDS fizeram referência ao trabalho de Goffman (1963) para definir estigma internalizado.

No estudo de Sayles, Hays, Sarkisian, Mahajan e Spritzer (2008), os próprios autores definiram o conceito a partir da conceituação do processo de estigma social. Segundo eles, o estigma internalizado ocorre quando um indivíduo internaliza normas culturais e narrativas que o identificam como membro de um grupo desviante e assume a "identidade deteriorada", como descrito no trabalho de Goffman (1963). Kalichman et al. (2009) também apresentaram a clássica teoria do modelo de estigma social descrita por Goffman (1963), a qual, segundo os autores, identifica três dimensões do estigma que também são características do HIV/AIDS: caráter pessoal marcado, identidade social marcada e deformidades ou defeitos físicos.

Excluindo-se os dois casos anteriormente citados, o autor mais referenciado na conceituação do estigma internalizado foi Patrick Corrigan, citado em oito estudos (Ritsher et al., 2003; Corrigan et al., 2006; Vogel et al., 2006; Ersoy \& Varan, 2007; Fung et al., 2007; Kanter et al., 2008; Rao et al., 2009; Barney, Griffiths, Christensen, \& Jorm, 2010). Para esse autor, a internalização do estigma ocorre à medida que o indivíduo torna-se consciente dos estereótipos negativos que as outras pessoas endossam (consciência de estereótipos), concorda pessoalmente com esses estereótipos (concordância com estereótipos) e os aplica a si mesmo, ocasionando a diminuição da autoestima (Corrigan, 1998; Corrigan \& Watson, 2002; Corrigan, 2004; Corrigan et al., 2006).

Lillis, Luoma, Levin \& Hayes (2010) usaram a definição proposta por Link e Phelan (1998), segundo os quais o estigma internalizado se inicia quando as pessoas desenvolvem teorias a respeito dos pacientes com transtornos mentais a partir dos conhecimentos que refletem a imagem cultural destes transtornos. Por sua vez, os pacientes com transtornos mentais internalizam esses conhecimentos estereotipados e antecipam o preconceito e a discriminação que poderão vir a sofrer. Por fim, Vogel et al., (2006) citaram, além de outros autores, Holmes e River (1998), para apresentar algumas consequências do estigma internalizado. Para os autores, o autoestigma resulta em perda da autoestima, em diminuição da auto-eficácia e em maior resistência à participação nas interações sociais (Holmes \& River, 1998).

\section{Principais justificativas apresentadas pelos autores para a construção/validação das escalas}

Diante da variedade de justificativas apresentadas pelos autores para a construção e/ou validação das escalas, duas merecem destaque. A primeira - mais utilizada - foi a falta de escalas para medir o estigma internalizado em relação a condições específicas (Barney et al., 2010; Ersoy \& Varan, 2007; Fung et al., 2007; Kalichman et al., 2009; Kanter et al., 2008; Lillis et al., 2010; Rao et al., 2009; Sayles et al., 2008; Vogel et al., 2006).

A segunda justificativa apresentada foi a importância de se avaliar o estigma internalizado, uma vez que este influencia diretamente o tratamento dos pacientes. Assim, para os autores que utilizaram essa justificativa, a construção/validação desse tipo de escala pode contribuir para o desenvolvimento de estratégias de redução de estigma internalizado e de seus efeitos negativos, estimular a adesão ao tratamento, assim como melhorar os resultados de recuperação dos pacientes (Corrigan et al., 2006; Fung et al., 2007; Ritsher et al., 2003; Vogel et al., 2006). 


\section{Aspectos metodológicos dos estudos}

No que diz respeito aos aspectos metodológicos dos estudos, foram analisados a população e os instrumentos utilizados em cada estudo. No tocante à população investigada, quatro estudos utilizaram amostras de pacientes com transtornos mentais gerais: Ritsher et al. (2003) investigaram uma amostra de 127 pacientes; no estudo de Corrigan et al. (2006), o grupo amostral foi composto por 60 participantes; a amostra do estudo de Fung et al. (2007) foi composta por 108 participantes e, Ersoy e Varan (2007) investigaram 203 pacientes.

As amostras dos dois estudos voltados à construção das escalas de estigma internalizado da depressão não foram compostas estritamente por pacientes com diagnóstico de depressão. Kanter et al. (2008) utilizaram uma amostra de 391 participantes divididos em dois grupos: um grupo de estudantes de graduação $(n=168)$ e um grupo de membros da comunidade geral $(n=223)$. Quanto ao histórico de depressão, no grupo de estudantes, 25,8\% dos participantes expuseram uma história de terapia e medicação com antidepressivos; já entre os participantes do grupo de membros da comunidade, essa porcentagem foi de 53,9\%. No estudo de Barney et al. (2010), na aplicação da versão final da SelfStigma of Depression Scale (SSDS) utilizou-se uma amostra de 330 participantes, todos membros de uma rede da internet sobre iniciativas para a depressão, em que $86 \%$ dessas pessoas relataram ter tido experiência pessoal de depressão.

Para a construção de escalas de mensuração do estigma internalizado de portadores do HIV/AIDS, a população estudada constituiu-se de portadores desta condição. A amostra de Sayles et al. (2008) continha 202 pessoas portadoras de HIV, enquanto Kalichman et al. (2009) utilizaram uma amostra de três diferentes países: África do Sul $(n=1068)$, Suazilândia $(n=1090)$ e Estados Unidos $(n=239)$.

Os outros estudos utilizaram amostras bem diversificadas: Vogel et al. (2006), para mensurar o estigma internalizado na busca por ajuda psicológica, utilizaram uma amostra total de 1.816 estudantes universitários, dividida em cinco estudos. A amostra do estudo de Rao et al. (2009) foi composta de 511 participantes portadores de transtornos neurológicos. Por fim, no estudo de Lillis et al. (2010), 62 pessoas consideradas obesas, a partir dos critérios propostos pelos autores, responderam ao questionário de estigma internalizado relacionado ao peso.

Em relação aos instrumentos, estes foram utilizados nos artigos com o objetivo principal de verificar as propriedades psicométricas da escala construída/validada em questão. Todos os artigos analisados utilizaram mais de um instrumento como estratégia metodológica para a consolidação do estudo. $\mathrm{O}$ escore total de instrumentos utilizados entre os 11 artigos analisados foi de 60 instrumentos, dos quais apenas cinco se repetiram entre os estudos.

A Rosemberg Self-Esteem Scale esteve presente em 6 estudos (Barney et al., 2010; Corrigan et al., 2006; Ersoy \& Varan, 2007; Fung et al., 2007; Ritsher et al., 2003; Vogel et al., 2006). A Center for Epidemiologic Studies Depression Scale (CES-D) foi utilizada em três estudos (Kalichman et al., 2009; Kanter et al., 2008; Ritsher et al., 2003). O Brief Symptom Inventory (BSI) foi empregado em dois artigos (Ersoy \& Varan, 2007; Lillis et al., 2010), assim como a Devaluation Discrimination Scale (Corrigan et al., 2006; Ritsher et al., 2003) e a SelfEfficacy Scale (Corrigan et al., 2006; Fung et al., 2007).

\section{Procedimentos utilizados na construção e validação das escalas}

Os procedimentos utilizados pelos autores para a realização da validação ou construção de uma escala foram diferentes, por isso optou-se por dividir essa sessão em duas categorias distintas: validação e construção.

\section{Validação}

Os dois estudos voltados à validação das escalas de estigma internalizado de transtorno mental realizaram procedimentos semelhantes. Para validação da versão turca da Internalized Stigma of Mental Illness (ISMI), dois psiquiatras e um psicólogo clínico participaram da tradução da escala. Esses três tradutores avaliaram a correção, a clareza e o entendimento das diferentes traduções de cada item e decidiram a tradução final. O termo "doença psiquiátrica" foi utilizado em preferência ao termo "doença mental", uma vez que este foi considerado pelos autores como mais estigmatizante que aquele (Ersoy \& Varan, 2007).

Já no processo de validação da versão chinesa da Self-Stigma of Mental Illness Scale (SSMIS), um tradutor qualificado traduziu os itens da escala do inglês para o chinês para que os respondentes da língua cantonesa pudessem entender a escala. Posteriormente, a versão chinesa traduzida da escala foi retrotraduzida independentemente para a língua inglesa também por um tradutor capacitado. As discrepâncias semânticas foram identificadas por dois pós-graduados experientes em pesquisa em saúde 
mental e avaliadas por dois pesquisadores doutores experientes em reabilitação psiquiátrica. As pequenas alterações feitas na versão chinesa incluíram adaptação de alguns itens e correção de algumas palavras traduzidas (Fung et al., 2007).

\section{Construção}

Em relação à construção das escalas, os procedimentos mais adotados para a composição dos itens da nova escala foram a utilização de outras escalas e a condução de grupos focais, ambos empregados por seis estudos. Cinco estudos ainda utilizaram revisão da literatura, quatro, um comitê de especialistas e dois, a condução de entrevistas cognitivas na construção das escalas. $\mathrm{O}$ estudo que utilizou todos esses procedimentos foi o de Rao et al. (2009) e os que utilizaram um único procedimento foram Vogel et al. (2006) e Kalichman et al. (2009), apresentados na Tabela 01 .

Tabela 1. Procedimentos utilizados para a composição dos itens das escalas.

\begin{tabular}{|c|c|c|c|c|c|}
\hline Sigla da escala & $\begin{array}{c}\text { Utilização de outras } \\
\text { escalas }\end{array}$ & Grupos focais & Revisão da literatura & Comitê de especialistas & Entrevistas cognitivas \\
\hline$\overline{\mathrm{ISMI}}$ & ( & $\mathrm{x}$ & & & \\
\hline SSMIS & $\mathrm{x}$ & $\mathrm{x}$ & & & \\
\hline SSOSH & & & & $\mathrm{x}$ & \\
\hline DSSS & $\mathrm{x}$ & & $\mathrm{x}$ & $\mathrm{x}$ & \\
\hline IHSS & & $\mathrm{x}$ & $\mathrm{x}$ & & $\mathrm{x}$ \\
\hline IA-RSS & $\mathrm{x}$ & & & & \\
\hline SSCI & $\mathrm{x}$ & $\mathrm{x}$ & $\mathrm{x}$ & $\mathrm{x}$ & $\mathrm{x}$ \\
\hline SSDS & & $\mathrm{x}$ & $\mathrm{x}$ & & \\
\hline WSSQ & $\mathrm{x}$ & $\mathrm{x}$ & $\mathrm{x}$ & $\mathrm{x}$ & \\
\hline
\end{tabular}

\section{Propriedades Psicométricas das escalas}

Somente três estudos (Rao et al., 2009; Sayles et al., 2008; Vogel et al., 2006) explicitaram como se deu a avaliação da validade de conteúdo das propriedades psicométricas das escalas. Os autores dos outros oito estudos não explicitaram se a propriedade foi avaliada, nem como se deu o processo. Dos onze estudos analisados, apenas dois (Kalichman et al., 2009; Vogel et al., 2006) avaliaram a validade de critério. Vogel et al. (2006) compararam a Self-Stigma of Seeking Help (SSOSH) com outros testes, enquanto Kalichman et al. (2009) utilizaram a comparação entre grupos (verificou a diferença nos escores da Internalized AIDS-Related Stigma Scale - IA-RSS entre indivíduos que relataram ter tido experiência de discriminação e indivíduos que relataram não ter tido experiências de discriminação) para avaliar a validade de critério.

Todas as medidas, exceto a SSDS (Barney et al., 2010), apresentaram a validade de constructo com resultados adequados. Apesar de a análise fatorial ter indicado e correlacionado os quatro fatores da SSDS (Barney et al., 2010) e a escala ter apresentado uma boa consistência interna $(\alpha=$
0,87 ), as associações entre a SSDS e todas as outras escalas utilizadas para avaliar a validade de constructo foram fracas, sendo que, em alguns casos, não houve correlações.

Com exceção da Stigma Scale for Chronic Illness - SSCI (Rao et al., 2009), todas as medidas avaliaram a confiabilidade das escalas apresentando resultados satisfatórios de consistência interna. Rao et al. (2009) apresentaram o valor da consistência interna referente à escala de 26 itens, no entanto, a SSCI final possui 24 itens no total.

Dos quatro estudos que não realizaram o testereteste para também avaliar a confiabilidade das escalas (Ersoy \& Varan, 2007; Kanter et al., 2008; Rao et al., 2009; Sayles et al., 2008), apenas um (Ersoy \& Varan, 2007) utilizou outro método em substituição: o método das metades, também conhecido como "Split-half". Dos sete estudos que realizaram o teste-reteste, somente um (Kalichman et al., 2009) apresentou os valores do $\alpha$ de Cronbach abaixo de 0,70. A confiabilidade do teste-reteste na Cidade do Cabo e em Atlanta foram, respectivamente: $\alpha=0,45 ; \alpha=0,62$ (Kalichman et al., 2009). 
Tabela 2. Avaliação das propriedades psicométricas das Escalas de Estigma Internalizado.

\begin{tabular}{|c|c|c|c|c|c|}
\hline Sigla da escala & Validade de Conteúdo ${ }^{1}$ & $\begin{array}{l}\text { Validade de } \\
\text { Critério }^{2}\end{array}$ & Validade de Constructo ${ }^{3}$ & Consist. Interna $^{4}$ & Teste-reteste ${ }^{5}$ \\
\hline ISMI & $?$ & $\mathrm{x}$ & + & + & + \\
\hline SSMIS & $?$ & $\mathrm{x}$ & + & + & + \\
\hline SSOSH & + & + & + & + & + \\
\hline $\begin{array}{l}\text { Turkish } \\
\text { ISMI }\end{array}$ & $?$ & $\mathrm{x}$ & + & + & $\begin{array}{c}+ \\
(\text { Split-half })\end{array}$ \\
\hline CSSMIS & $?$ & $\mathrm{x}$ & + & + & + \\
\hline DSSS & $?$ & $\mathrm{x}$ & + & + & $\mathrm{x}$ \\
\hline IHSS & + & $\mathrm{x}$ & + & + & $\mathrm{x}$ \\
\hline IA-RSS & $?$ & + & + & + & - \\
\hline SSCI & + & $\mathrm{x}$ & + & 0 & $\mathrm{x}$ \\
\hline SSDS & $?$ & $\mathrm{x}$ & - & + & + \\
\hline WSSQ & $?$ & $\mathrm{x}$ & + & + & + \\
\hline
\end{tabular}

$\mathrm{x}=$ propriedade não foi avaliada;

$?=$ os autores não explicitaram se a propriedade foi avaliada, nem como se deu o processo.

Foram usadas para verificação da validade de conteúdo: análise de juízes experientes na área; grupos focais/entrevistas cognitivas.

Foram usadas para verificação da validade de critério: comparação com outros testes; comparação entre grupos, sendo p < 0,001 .

3 Foram usadas para verificação da validade de constructo: relação com outros testes (hipóteses específicas foram formuladas e até 75\% dos resultados estavam de acordo com essas hipóteses, sendo $\mathrm{p}<0,001)$; verificação da consistência interna dos itens; análise fatorial (a análise apresentou um total de itens dentro dos fatores esperados).

$4 \quad$ Alpha de Cronbach maior ou igual a 0.7.

5 Alpha de Cronbach ou índice Kappa maior ou igual a 0.7.

$+=$ índice positivo da propriedade;

- = índice negativo da propriedade;

$0=$ resultado não foi apresentado;

Apesar das limitações dos estudos principalmente no que diz respeito à representatividade das amostras - de acordo com as propriedades psicométricas apresentadas, todos os autores das escalas construídas/validadas concluíram que estas podem ser consideradas instrumentos úteis na mensuração do estigma internalizado.

\section{DISCUSSÃO}

A literatura preconiza que, quando não se dispõe de um instrumento de medida adaptado e validado para uso na cultura onde se vive, pode-se desenvolver um novo instrumento de medida ou adaptar um instrumento previamente validado em outra linguagem (Guillemin, Bombardier, \& Beaton, 1993). Nesta revisão, obtiveram-se dois estudos de validação e nove de construção de instrumentos para mensurar estigma internalizado. Este pode ser considerado um resultado positivo, uma vez que, apesar dos estudos de validação de instrumentos minimizarem tempo e custos e tornarem possível a comparação de resultados em estudos multicêntricos, eles muitas vezes são criticados porque a versão criada não é, ao mesmo tempo, equivalente com o instrumento original, nem tampouco compreensível para a população-alvo daquele país.

$\mathrm{O}$ reduzido número de estudos de validação de escalas encontrados nesta revisão também vai ao encontro da justificativa apresentada pelos autores como motivo da construção das escalas, ou seja, a falta de instrumentos válidos para mensuração do estigma internalizado; no entanto esse argumento não se justifica ao analisarmos de perto as características das populações dos estudos, tanto no que se refere ao tipo de transtorno relacionado ao estigma internalizado, quanto ao país do estudo.

Em relação ao tipo do transtorno, os resultados encontrados apontam que há uma variedade de escalas para mensuração do estigma internalizado de determinadas condições de saúde, no entanto há uma ênfase nos transtornos mentais. Devem ser realizadas investigações que busquem identificar o impacto do estigma internalizado em outras populações que não somente as pessoas com transtornos mentais, uma vez que as diferenças entre as condições estigmatizadas podem influenciar de diversas maneiras as variáveis 
relevantes para o processo de internalização do estigma.

Esta ênfase deve ser refletida também na medida em que existem não só escalas gerais, mas também específicas para transtornos mentais, como, por exemplo, para a depressão. Cabe questionar as razões para tanto, isto é, se serão criadas tantas escalas quantos forem os transtornos mentais diagnosticáveis, ou se as escalas gerais não são generalizáveis o bastante para mensurar o estigma internalizado em relação aos diversos transtornos e por isso devem ser substituídas.

Outro ponto a ser observado é a discordância de alguns autores quando se trata da avaliação do estigma internalizado de um mesmo transtorno. Na presente revisão, foram analisados dois artigos voltados à construção de escalas de estigma internalizado de transtorno mental, depressão e HIV/AIDS. Visto que os autores dos primeiros estudos os justificam pela ausência de escalas construídas, cabe analisar os autores dos estudos subsequentes. Corrigan et al. (2006) não justificam o porquê da construção de uma nova escala de estigma internalizado de transtorno mental, nem mesmo cita o trabalho de Ritsher et al. (2003), assim como Kalichman et al. (2009) não citam a escala desenvolvida por Sayles et al. (2008) para a mensuração do estigma internalizado voltado para pessoas com HIV/AIDS. Kalichman et al. (2009) argumentam apenas que as medidas anteriores de estigma internalizado da AIDS têm enfatizado as ramificações sociais da revelação do HIV e as reações públicas quanto à AIDS, de forma que essas pesquisas não estão voltadas à avaliação do estigma internalizado.

A falta de compreensão entre os autores quanto a que, de fato, a escala esteja medindo foi a principal justificativa apresentada por Barney et al. (2010) para a construção da escala de estigma internalizado da depressão. De acordo com Barney et al. (2010), as pesquisas não têm apresentado uma demarcação clara entre os elementos de estigma internalizado, estigma percebido e estigma público. Os autores citam que um grupo desenvolveu a Depression Self-Stigma Scale DSSS (Kanter et al., 2008), no entanto, o foco dessa escala é a percepção do estigma. Assim, dentre os vários motivos que levariam diferentes autores a construir escalas sobre os mesmos transtornos, poderíamos destacar a confusão conceitual quanto à definição de estigma internalizado. Apesar de o sentido ser o mesmo, Corrigan et al. (2006) propõem um conceito de estigma internalizado mais voltado para sua relação com a autoestima e a autoeficácia, diferentemente do proposto por Ritsher et al. (2003), que destacam a desvalorização, a vergonha, o sigilo e a exclusão no processo de internalização do estigma. Kalichman et al. (2009) e Sayles et al. (2008) tentam usar o trabalho de Goffman (1963) sobre estigma público, mas com abordagens diferentes.

Segundo Link e Phelan (2001), as várias definições conceituais relacionadas ao processo de estigmatização, assim como a falta de clareza das mesmas, dificultam a comparação de resultados entre os estudos. As diferentes operacionalizações dos conceitos, somadas à falta de especificidade das medidas e construtos utilizados, impossibilitam a construção de sínteses que evidenciem a influência do estigma na vida das pessoas (Link \& Phelan, 2001).

Em uma recente revisão da literatura realizada por Brohan et al. (2010) tendo como objetivo analisar as medidas de estigma, preconceito e discriminação contra pessoas com transtornos mentais, os autores expuseram a dificuldade que tiveram em categorizar o estigma em estigma percebido, expeiência de estigma e estigma internalizado. Segundo os autores, essas distinções foram úteis para organizar a revisão, porém as várias interconexões entre os conceitos dificultava, na maior parte do tempo, a decisão quanto a qual conceito seria mais adequado para qualificar uma subescala (Brohan et al., 2010). Estes pontos reforçam a natureza complexa do conceito de estigma.

Já no que se refere aos países de realização dos estudos, sete estudos de construção de escalas foram realizados especificamente nos EUA. Esses dados confirmam a carência de desenvolvimento de instrumentos em outros países que não seja os EUA. Somado isto ao problema da repetição da construção de escalas de estigma internalizado para os mesmos transtornos, no caso dos estudos de Corrigan et al. (2006) e Ritsher et al. (2003) haveria ainda menos razões para a criação de outra escala, na medida em que ambos foram realizados nos EUA - o que não ocorre com os estudos de Sayles et al. (2008) e Kalichman et al. (2009), e Kanter et al. (2008) e Barney et al. (2010). No que se refere à validação, a ISMI (Ritsher et al., 2003) foi validada na Turquia e na China, porém o artigo de validação estava escrito em língua chinesa, o que nos impossibilitou de incluílo na revisão. Podemos notar, ainda, que a SSMIS (Corrigan et al., 2006) também foi validada na China por Fung et al. (2007).

Quanto aos problemas metodológicos apresentados pelas pesquisas, em relação à amostra, os estudos de Kanter et al. (2008) e Barney et al. (2010) não foram realizados em pacientes com diagnóstico de depressão. De acordo com Noronha e Baptista (2007) "a AERA, APA e NCME (1999) recomendam que, 
quando se realizam estudos de validade, deve-se definir claramente a população à qual o teste é destinado, poid (...) nenhum teste é válido para todos os propósitos ou para todas as situações" (p. 55). Não há padronização no emprego dos instrumentos utilizados, uma vez que 60 instrumentos foram utilizados, ao todo, nos 11 estudos analisados. Outra falha apresentada em três estudos foi a omissão dos itens que compõem as escalas. Apesar de Ersoy e Varan (2007) não terem apresentado a versão turca dos itens da ISMI (Ritsher et al., 2003), estes são conhecidos - em sua versão original - através do estudo de Ritsher et al. (2003). No entanto, Corrigan et al. (2006) não apresentaram os itens finais da escala SSMIS (Corrigan et al., 2006), assim como os mesmos itens não foram apresentados em sua versão chinesa no estudo de Fung et al. (2007), o que nos impossibilitou de verificar se os itens da escala SSMIS (Corrigan et al., 2006) possuem semelhanças com a ISMI (Ritsher et al., 2003). Visto isso, é de fundamental importância que os investigadores especifiquem o que estão mensurando e de que forma o estão fazendo, discutindo as implicações dos resultados.

A falta de padronização nos procedimentos utilizados pelos autores para construção e validação das escalas pode ser um indicador da necessidade de especialistas em construção/validação de escalas acompanhando o estudo sobre estigma internalizado. Dos onze estudos analisados, apenas o de Rao et al. (2009) utilizou um "guideline" para a construção da escala. Apesar de haver grupos e linhas de pesquisa em diversos países que estudam os fatores envolvidos no processo de estigmatização, faz-se necessária a consolidação de grupos com um maior amadurecimento teórico e instrumental sobre a mensuração do estigma internalizado.

Por fim, em relação às propriedades psicométricas das escalas, encontramos dificuldades em categorizá-las, uma vez que, além de existirem vários tipos de classificação quanto às evidências de validade, os autores não explicitavam qual definição eles estavam utilizando. O principal ponto a dificultar o processo de categorização das evidências de validade foi a validade de conteúdo. Segundo Noronha e Baptista (2007), para validação do conteúdo geralmente se recorre a um comitê de juízes especialistas na área; no entanto, houve estudos que recorreram à análise de juízes, mas não explicitaram se a validade de conteúdo foi avaliada, e vice-versa. Assim, optamos por incluir na tabela de análise das propriedades psicométricas das escalas apenas aqueles estudos que deixaram explícito que esta evidência de validade foi avaliada. Um pesquisador que se propõe a estudar a construção de escalas deve estar ciente de que o delineamento de uma pesquisa exige um conhecimento aprofundado não só do constructo pesquisado, mas também das qualidades psicométricas dos instrumentos; assim, para que se possa ter clareza sobre as reais condições e possibilidades de medidas dessas escalas é fundamental o conhecimento sobre sua qualidade (Noronha \& Baptista, 2007).

Apesar das limitações inerentes às escolhas metodológicas adotadas nesta revisão, a sistematização do processo permite que este estudo seja replicado no futuro, desde que se conte com outras delimitações temporais que permitam comparações com os resultados aqui apresentados. Dessa forma, considerando o pequeno número de estudos analisados, sugere-se uma revisão futura que abarque outras fontes, inclusive em outros idiomas.

\section{CONSIDERAÇÕES FINAIS}

Constamos que os estudos levantados nessa revisão voltados à construção/validação de instrumentos de mensuração do estigma internalizado apresentam uma série de significativos problemas conceituais e metodológicos. Além disso, estes estudos não vêm se propagando em relação a diferentes transtornos, tampouco em diversos países, de forma que os transtornos mentais são os mais pesquisados. Do mesmo modo, o país onde mais se desenvolvem e publicam estudos sobre construção de escalas de estigma internalizado são os EUA. No caso específico do Brasil, não há estudos de construção nem de validação de escalas de estigma internalizado, o que limita a realização de estudos sobre o tema no país.

Apesar desses indicadores negativos, deve-se destacar que o crescente número de escalas demonstra a relevância do tema nas diferentes áreas - inclusive na saúde mental - e proporciona um avanço do conhecimento à medida que investiga os fatores envolvidos no processo de estigmatização. Assim, fazse necessário um maior investimento nesse campo de pesquisa, uma vez que entre as diversas contribuições da utilização de instrumentos na área da saúde estão o auxílio no diagnóstico e na avaliação da eficácia do tratamento, possibilitando melhor direcionamento de recursos (Costa et al., 2009). 


\section{REFERÊNCIAS}

Barney, L. J., Griffiths, K. M., Christensen, H., \& Jorm, A. F. (2010). The Self-Stigma of Depression Scale (SSDS): development and psychometric evaluation of a new instrument. International Journal of Methods in Psychiatric Research, 19(4), 243-254.

Barney, L. J., Griffiths, K. M., Jorm, A. F., \& Christensen, H. (2006). Stigma about depression and its impact on help-seeking intentions. Australian and New Zealand Journal of Psychiatry, 40(1), 51-54.

Brohan, E., Slade, M., Clement, S., \& Thornicroft, G. (2010). Experiences of mental illness stigma, prejudice and discrimination: a review of measures. BMC Health Services Research, 10(80), 1-11.

Corrigan, P. W. (1998). The Impact of Stigma on Severe Mental Illness. Cognitive and Behavioral Practice, 5, 201-222.

Corrigan, P. W. (2004). How Stigma Interferes With Mental Health Care. American Psychologist, 59(7), 614-625.

Corrigan, P. W., \& Watson, A. C. (2002). The paradox of self-stigma and mental illness. Clinical Psychology: Science and Practice, 9(1), 35-53.

Corrigan, P. W., Watson, A. C., \& Barr, L. (2006). The self-stigma of mental illness: Implications for selfesteem and self-efficacy. Journal of Social and Clinical Psychology, 25(8), 875-884.

Costa, F. S., Bandeira, D. R., Trentini, C., Brilmann M., Friedman, R., \& Nunes, M. A. (2009). Considerações acerca da avaliação psicológica das comorbidades psiquiátricas em obesos. Psicologia em Estudo, 14(2), 287-293.

Crocker, J., \& Lawrence, J. S. (1999). Social stigma and self-esteem: the role of contingencies of worth. In D. A. Prentice \& D. T. Miller (Org.), Cultural divides: Understanding and overcoming group conflict (pp.364392). New York: Russel Sage Foundation.

Crocker, J., \& Major, B. (1989). Social stigma and selfesteem: the self-protective properties of stigma. Psychiatry Research, 96(4), 608-630.

Ersoy, M. A., \& Varan, A. (2007). Reliability and validity of the Turkish version of the internalized stigma of mental illness scale. Turkish Journal of Psychiatry, 18(2), 163-171.

Fung, K. M. T., Tsang, H. W. H., Corrigan, P. W., Lam, C. S., \& Cheng, W. M. (2007). Measuring self-stigma of mental illness in China and its implications for recovery. International Journal of Social Psychiatry, 53(5), 408-418.

Goffman, E. (1963). Notes on the Management of a Spoiled Identity. New York: Simon \& Schuster.

Guillemin, F., Bombardier, C., \& Beaton, D. (1993). Crosscultural adaption of health-related quality of life measures: literature review and proposed guidelines. Journal of Clinical Epidemiology, 46(12), 1417-1432.
Holmes, E. P., \& River, L. P. (1998). Individual strategies for coping with the stigma of severe mental illness. Cognitive and Behavioral Practice, 5(2), 231-239.

Kalichman, S. C., Simbayi, L. C., Cloete, A., Mthembu, P. P., Mkhonta, R. N., \& Ginindza, T. (2009). Measuring AIDS stigmas in people living with HIV/AIDS: The Internalized AIDS-Related Stigma Scale. AIDS Care, 21(1), 87-93.

Kanter, J. W., Rusch, L. C., \& Brondino, M. J. (2008). Depression self-stigma - A new measure and preliminary findings. Journal of Nervous and Mental Disease, 196(9), 663-670.

Lillis, J., Luoma, J. B., Levin, M. E., \& Hayes, S. C. (2010). Measuring weight self-stigma: the weight selfstigma questionnaire. Obesity (Silver Spring), 18(5), 971-6.

Link, B. G., \& Phelan, J. C. (2001). Conceptualizing Stigma. Annual Review of Sociology, 27, 363 - 385.

Link, B. G., Mirotznik, J., \& Cullen, F. T. (1991). The Effectiveness of Stigma Coping Orientations: Can Negative Consequences of Mental Illness Labeling be Avoided? Journal of Health and Social Behavior, 32, 302-320.

Link, B. G., Phelan, J. C., Bresnahan, M., Stueve, A., \& Pescosolido, B. A. (1999). Public Conceptions of Mental Illness: Labels, Causes, Dangerousness, and Social Distance. American Journal of Public Health, 89(9), 1328-1333.

Link, B. G., Struening, E. L., Neese-Todd, S., Asmussen, S., \& Phelan, Jo C. (2001). The Consequences of Stigma for the Self-Esteem of People With Mental Illness. Psychiatric Services, 52(12), 1621-1626.

Luoma, J. B., Kohlenberg, B. S., Hayes, S. C., Bunting, K., \& Rye, A. K. (2008). Reducing self-stigma in substance abuse through acceptance and commitment therapy: Model, manual development, and pilot outcomes. Addiction Research \& Theory, 16(2), 149-165.

Noronha, A. P. P., \& Baptista, M. N. (2007). Relação entre metodologia e avaliação psicológica. In M. N. Baptista \&, D. C. Campos (Orgs.). Metodologias de pesquisa em ciências: análises quantitativa e qualitativa (pp. 4960). Rio de Janeiro: LTC.

Rao, D., Choi, S. W., Victorson, D., Bode, R., Peterman, A., Heinemann, A., et al. (2009). Measuring stigma across neurological conditions: The development of the Stigma Scale for Chronic Illness (SSCI). Quality of Life Research: An International Journal of Quality of Life Aspects of Treatment, Care \& Rehabilitation, 18(5), 585-595.

Ritsher, J. B., Otilingam, P. G., \& Grajales, M. (2003). Internalized stigma of mental illness: psychometric properties of a new measure. Psychiatry Research, 121(1), 31-41.

Ronzani, T. M., Furtado, E. F., \& Higgins-Biddle, J. (2009). Stigmatization of alcohol and other drug users by primary care providers in Southeast Brazil. Social Science \& Medicine, 69(7), 1080-1084. 
Sayles, J. N., Hays, R. D., Sarkisian, C. A., Mahajan, A. P., Spritzer, K. L., \& Cunningham, W. E. (2008). Development and psychometric assessment of a multidimensional measure of internalized HIV stigma in a sample of HIV-positive adults. AIDS and Behavior, 12(5), 748-758.

Aceito em 30/11/2011

Vogel, D. L., Wade, N. G., \& Haake, L. (2006). Measuring the Self-Stigma Associated With Seeking Psychological Help. Journal of Counseling Psychology, 53(3), 325-337.

Endereço para correspondência:
Rhaisa Gontijo Soares. Rua São João, $n^{\circ}$ 26, Centro, CEP 35500-043, Divinópolis-MG E-mail: rhaisags@yahoo.com.br. 